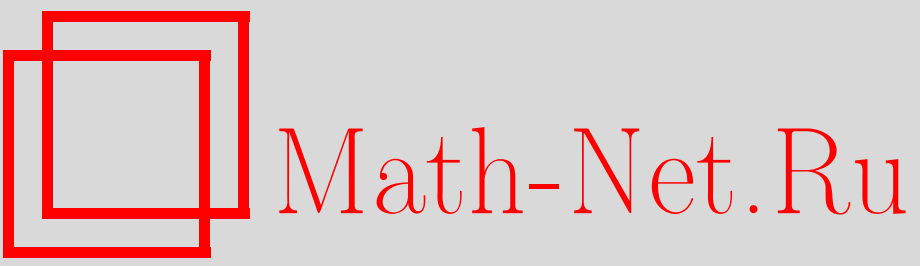

А. И. Перов, О теореме Брауэра и овалах Кассини, УМН, 1998, том 53, выпуск $1,225-226$

DOI: https://doi.org/10.4213/rm28

Использование Общероссийского математического портала Math-Net.Ru подразумевает, что вы прочитали и согласны с пользовательским соглашением

http://www.mathnet.ru/rus/agreement

Параметры загрузки:

IP : 54.198 .67 .100

26 апреля 2023 г., 18:21:48 


\section{О ТЕОРЕМЕ БРАУЭРА И ОВАЛАХ КАССИНИ}

\section{А. И. ПЕРОВ}

При изучении устойчивости линейных дискретных и непрерьвных систем важную роль играют различные локализационные теоремы [1], [2]. Пусть $A=\left(a_{j k}\right)$ - произвольная комплексная квадратная $(n \times n)$-матрица. Из указанных выше теорем можно получить как оценку спектрального радиуса $\operatorname{spr} A$ (наиболшшего из модулей собственных значений), так и оценку спектральной абсциссы $\operatorname{spa} A$ (наиболшшей из вещественных частей собственных значений).

Суммы модулей внедиагональных элементов $j$-й строки и $j$-го столбца обозначим, соответственно,

$$
p_{j}=\sum_{k \neq j}\left|a_{j k}\right|, \quad q_{j}=\sum_{k \neq j}\left|a_{k j}\right|, \quad j=1, \ldots, n .
$$

По теореме Гершгорина и Островского при любом фиксированном $\alpha, 0 \leqslant \alpha \leqslant 1$, каждое собственное значение матрицы $A$ лежит по крайней мере в одном из $n$ кругов

$$
\left|\lambda-a_{j j}\right| \leqslant p_{j}^{1-\alpha} q_{j}^{\alpha}, \quad j=1, \ldots, n,
$$

с центрами в точках $a_{j j}$ и радиусами $p_{j}^{1-\alpha} q_{j}^{\alpha}$. При $\alpha=0$ и $\alpha=1$ получаем круги Гершгорина, построенные по строкам и столбцам матрицы $A$ соответственно, а при $0<\alpha<1$ мы приходим к кругам Островского. Попутно отметим, что в книге [3, с. 239 , задача 25] ошибочно утверждается, что каждое собственное значение матрицы располагается в некотором круге с центром в точке $a_{j j}$ и радиусом $\min \left(p_{j}, q_{j}\right)$ (т.е. пересечение объединений заменено объединением пересечений, что неправомочно). Так, например, собственные значения $\lambda_{1} \approx 5,37$ и $\lambda_{2} \approx-0,37$ матрицы с элементами $a_{11}=a_{12}=1, a_{21}=6, a_{22}=4$ не лежат ни в одном из кругов $|\lambda-1| \leqslant 1$ и $|\lambda-4| \leqslant 1$.

Из (2) вытекают оценки спектрального радиуса и спектральной абсциссы:

$$
\begin{aligned}
& \operatorname{spr} A \leqslant \max _{j}\left\{\left|a_{j j}\right|+p_{j}^{1-\alpha} q_{j}^{\alpha}\right\}, \\
& \operatorname{spa} A \leqslant \max _{j}\left\{\operatorname{Re} a_{j j}+p_{j}^{1-\alpha} q_{j}^{\alpha}\right\}
\end{aligned}
$$

(индекс $j$ пробегает все значения $1, \ldots, n$ ), наилучшее значение которых может быть достигнуто минимизацией по параметру $\alpha$.

Брауэру, а затем Островскому, удалось усилить сформулированный выше результат (см. (2)) и показать, что при любом фиксированном $\alpha, 0 \leqslant \alpha \leqslant 1$, каждое собственное значение матрицы $A$ лежит внутри или на границе по крайней мере одного из $n(n-1) / 2$ овалов Кассини

$$
\left|\lambda-a_{j j}\right|\left|\lambda-a_{k k}\right| \leqslant\left(p_{j} p_{k}\right)^{1-\alpha}\left(q_{j} q_{k}\right)^{\alpha} \equiv r_{j k}^{2}(\alpha), \quad j<k .
$$

При попыткеприменить указанную теорему для получения оценок спектрального радиуса и спектральной абсциссы мы сталкиваемся со следующими задачами на условньй экстремум. Пусть $a$ и $b$ - произвольные комплексные числа, $r$ - произвольное вещественное неотрицательное число. Требуется найти наибольшее значение модуля $|\lambda|$, а также вещественной части $\operatorname{Re} \lambda$, когда $\lambda$ пробегает овал

$$
|\lambda-a||\lambda-b|=r^{2} .
$$

$\mathrm{K}$ сожалению, мы не располагаем в общем случае точными аналитическими формулами для максимумов интересующих нас величин. Напомним, что точки $a$ и $b$ называются фокусами овала, а число $r$ - его радиусом.

Наши оценки основаны на замене овала (6) окружностью с центром в точке $(a+b) / 2$ и радиусом $s$, равным корню квадратному из суммы квадрата половины фокусного расстояния и квадрата радиуса овала, или әллипсом с фокусами в точках $a$ и $b$ и полуосямu $s$ и $r$. Полученные нами оценки позволяют сформулировать и доказать следующие утверждения, приводящие в ряде случаев к более точньм по сравнению с неравенствами (3) и (4) результатам. 
Теорема 1. Для спектрального радиуса матрищы $A$ при любом фиксированном $\alpha$, $0 \leqslant \alpha \leqslant 1$, справедливы оценки

$$
\begin{aligned}
& \operatorname{spr} A \leqslant \max _{j<k}\left\{\frac{\left|a_{j j}\right|+\left|a_{k k}\right|}{2}+\left[\left(\frac{\left|a_{j j}\right|-\left|a_{k k}\right|}{2}\right)^{2}+r_{j k}^{2}(\alpha)\right]^{1 / 2}\right\}, \\
& \operatorname{spr} A \leqslant \max _{j<k}\left\{\left|\frac{a_{j j}+a_{k k}}{2}\right|+\left[\left(\frac{\left|a_{j j}-a_{k k}\right|}{2}\right)^{2}+r_{j k}^{2}(\alpha)\right]^{1 / 2}\right\} .
\end{aligned}
$$

Если выражения, стоящие справа в (7) и (8), меньше единицы, то все собственные значения матрицы $A$ по модулю меньше единицы, и она является асимптотически устойчив ой (в смысле теории устойчивости дискретных систем).

Теорема 2. Для спектральной абсциссы матрищы $A$ при любом фиксированном $\alpha$, $0 \leqslant \alpha \leqslant 1$, справедливы оченки

$$
\begin{aligned}
& \text { spa } A \leqslant \max _{j<k}\left\{\operatorname{Re}\left(\frac{a_{j j}+a_{k k}}{2}\right)+\left[\left(\operatorname{Re}\left(\frac{a_{j j}-a_{k k}}{2}\right)\right)^{2}+r_{j k}^{2}(\alpha)\right]^{1 / 2}\right\}, \\
& \text { spa } A \leqslant \max _{j<k}\left\{\operatorname{Re}\left(\frac{a_{j j}+a_{k k}}{2}\right)+\left[r_{j k}^{2}(\alpha)-\left(\operatorname{Im}\left(\frac{a_{j j}-a_{k k}}{2}\right)\right)^{2}\right]^{1 / 2}\right\} .
\end{aligned}
$$

Последняя оченка установлена при дополнительном предположении, что диагональные әлементы имеют одинаковые вещественные части, а подкоренные выражсения неотрицательны.

Если выражения, стоящие справа в (9) и (10), отрицательны, то все собственные значения матрицы $A$ лежат в открытой левой полуплоскости, а она является асимптотически устойчивой (в смысле теории устойчивости непрерывных систем).

Оценка (7) принадлежит Брауэру, остальные оценки (8)-(10) являются новыми. Для достижения наилучшего резултата при использовании одной из формул (7)-(10) следует произвести еще минимизацию по параметру $\alpha$. В заключение отметим, что написанные вьше оценки (см. теоремы 1 и 2) могут быть полезными и при изучении устойчивости в критических случаях [4].

\section{СПИСОК ЛИТЕРАТУРЫ}

[1] Пароди М. Локализация характеристических чисел матриц и ее применение. М.: ИЛ, 1960. [2] Маркус М., Минк Х. Обзор по теории матриц и матричных неравенств. М.: Наука, 1972. [3] Данфорд Н., Шварц Дж. Т. Линейные операторы. Т. 2. М.: Мир, 1966. [4] Перов А. И. // Вестн. ВГУ, сер. 2, естеств. науки. 1996. № 2. 